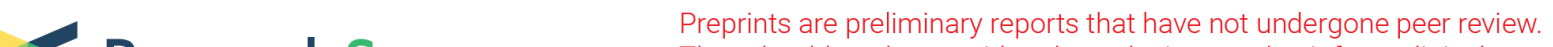 $\begin{array}{ll}\text { Research Square } & \text { They should not be considered conclusive, used to inform clinical practice, } \\ \text { or referenced by the media as validated information. }\end{array}$
}

\section{The Potential of Adipokines in Identifying Multiple Trauma Patients at Risk of Developing Multiple Organ Dysfunction Syndrome}

\section{Julian Haupt ( $\sim$ julianmirko@web.de )}

Klinikum rechts der Isar Experimental Trauma Surgery Ismaninger Strasse 2281675 Munich, Germany https://orcid.org/0000-0002-2608-3444

\section{Niels Krysiak}

Klinikum rechts der Isar der Technischen Universität München: Klinikum rechts der Isar der Technischen Universitat Munchen

\section{Marina Unger}

Klinikum rechts der Isar der Technischen Universität München: Klinikum rechts der Isar der Technischen Universitat Munchen

\section{Viktoria Bogner-Flatz}

Universitätsklinikum LMU München: Ludwig-Maximilians-Universitat Munchen

\section{Peter Biberthaler}

Klinikum rechts der Isar der Technischen Universität München: Klinikum rechts der Isar der Technischen Universitat Munchen

\section{Marc Hanschen}

Klinikum rechts der Isar der Technischen Universität München: Klinikum rechts der Isar der Technischen Universitat Munchen

\section{Martijn van Griensven}

Klinikum rechts der Isar der Technischen Universität München: Klinikum rechts der Isar der Technischen Universitat Munchen

\section{Alexander T. Haug}

Klinikum rechts der Isar der Technischen Universität München: Klinikum rechts der Isar der Technischen Universitat Munchen

\section{Research}

Keywords: Multiple trauma, Multiple organ dysfunction syndrome, Multiple organ failure, Adipokines, Leptin, Resistin, Interleukin-17A, Interleukin-33

Posted Date: October 16th, 2020

DOl: https://doi.org/10.21203/rs.3.rs-92157/v1 
License: (c) (i) This work is licensed under a Creative Commons Attribution 4.0 International License. Read Full License 


\section{Abstract}

\section{Background}

Multiple organ dysfunction syndrome (MODS) and the consecutive multiple organ failure (MOF) are severe and dreaded complications with a high mortality in multiple trauma patients. The aim of this study was to investigate the potential of the adipokines leptin, resistin, interleukin-17A and interleukin-33 as possible biomarkers in the early posttraumatic inflammatory response and for identifying severely traumatized patients at risk of developing MODS.

\section{Methods}

In total, 14 multiple trauma patients with an injury severity score (ISS) ${ }^{3} 16$ as well as a control group of 14 non-multiple trauma patients were included in this study and blood samples were taken at the time points $0,6,24,48$ and $72 \mathrm{~h}$ after admission. For the trauma patients, the SIRS and Denver MOF score were determined daily. The quantitative measurement of the plasma concentrations of the adipokines was performed using ELISA.

\section{Results}

In the statistical analysis, the multiple trauma patients showed statistically significant higher plasma concentrations of leptin, resistin, IL-17A and IL-33 compared to the control group. In addition, there was a statistically significant positive correlation between the concentrations of resistin, IL-17A and IL-33 and the corresponding SIRS scores and between the concentrations of resistin, IL-17A and IL-33 and the corresponding Denver MOF scores. Finally, ROC curve analysis revealed that the adipokines leptin and IL17A are suitable diagnostic markers for the discrimination between multiple trauma patients with and without MOF.

\section{Conclusions}

The results of this study may contribute to identify severely injured patients with a developing SIRS and MOF earlier, in order to adjust therapy strategies.

\section{Background}

Trauma represents the leading cause of death for people up to the age of 40 years in

Germany $[1,2]$. While overall mortality caused by severe trauma has decreased over the years, multiple organ dysfunction syndrome (MODS) and the consecutive multiple organ failure (MOF) are still severe complications with a high mortality [3-5]. MODS is caused by an imbalance or a dysregulation of the pro- and anti-inflammatory immune response [6, 7]. Characteristic for the pro-inflammatory immune response, the systemic inflammatory response syndrome (SIRS), is a local and systemic production of various mediators such as pro-inflammatory cytokines, complement factors, acute phase proteins and an 
accumulation of immune cells at the site of inflammation [6]. Adipokines are bioactive proteins that are produced by adipocytes and cells of the immune system [8, 9]. Adipokines exert their effect on metabolism, immunity and inflammation via an endocrine, paracrine and autocrine secretion mode [10].

The adipokine leptin is induced by TNF-a via the p55 TNF receptor in adipocytes and is a proinflammatory acute phase protein and a regulator of the fat metabolism $[8,11-13]$. Leptin exerts its proinflammatory properties via an activation and proliferation of monocytes and an increase in the production of TNF and IL-6 in the activated monocytes $[8,12,14]$. Leptin also induces chemotaxis and liberation of oxygen radicals from neutrophilic granulocytes [12]. On the other hand, leptin has also protective effects as shown in a two-hit trauma model [15].

The pro-inflammatory adipokine resistin is synthesized and secreted by adipocytes, endothelial cells, bone marrow cells and especially by mononuclear cells $[16,17]$. The synthesis is induced by the proinflammatory cytokines IL-1, IL-6 and TNF- $a$ as well as by lipopolysaccharides [18]. Resistin mediates its pro-inflammatory effect via the NF-KB signaling pathway and induces the synthesis of the proinflammatory cytokines IL-1 $\beta$, IL-6 and TNF-a in mononuclear cells [19].

The adipokine IL-17A is mainly secreted by $\mathrm{TH}-17$ cells and is induced by the pro-inflammatory cytokines IL-1, IL-6, IL-23 and TGF- $\beta$ [20-22]. IL-17A is a potent activator of neutrophil granulocytes and promotes their recruitment and migration [21, 22]. IL-17A also activates $T$ and $B$ cells, promotes priming of $T$ cells and antibody production as well as antibody class changes in B cells [20,23].

Interleukin-33 is expressed by a variety of cell types, including adipocytes, endothelial cells, fibroblasts and macrophages $[24,25]$. The release of IL-33 occurs mainly in the context of necrosis of cells, for example in a trauma or an infection and thus acts as an alarmin [26]. IL-33 acts as a chemoattractant and induces the production of the cytokines IL-5 and IL-13 in type 2 T helper cells [24, 27]. In addition, IL33 leads to the activation of eosinophilic granulocytes with an increased production of superoxide anions and IL-8, an induction of degranulation and an increase in cell survival [28]. Since the role of the adipokines leptin, resistin, interleukin-17A and interleukin-33 in trauma immunology has been poorly understood, the aim of this pilot study is to investigate the response of adipokines in the early posttraumatic systemic inflammatory immune response and whether adipokines are suitable diagnostic biomarkers to identify multiple trauma patients at risk of developing MODS in an intensive care setting.

\section{Methods}

\section{Patients}

The study was performed at our level 1 trauma center in accordance with the Good Clinical Practice Guidelines and the ethical standards as laid down in the 1964 Declaration of Helsinki and its later amendments. Ethical committee approval was obtained from the local institutional review board (reference number: 012/00). Signed informed consent was obtained from the patients or legal guardians. In this study, we included multiple trauma patients aged 18 and older with an injury severity score (ISS) of 
$\geq 16$. A total of 14 patients between the age of 19 and 79 (mean: 49.7 / SD \pm 19.2 ) years were enrolled in this study. The patient population consisted of six women and eight men. As a control group, we included 14 non-multiply traumatized individuals (eight women / six men), nine of whom received a simple plate osteosynthesis and five were completely healthy.

\section{Blood samples}

Four S-Monovettes EDTA $9 \mathrm{ml}$ (Sarstedt, Nümbrecht, Germany) blood samples were drawn from the multiple trauma patients on admission (not later than 90 minutes after trauma, indicated as $0 \mathrm{~h}$ ) and after 6, 24, 48 and 72 hours (h) at our intensive care unit. In total, 63 different blood samples could be obtained from the multiple trauma patients. From the control group, one S-Monovette EDTA $9 \mathrm{ml}$ (Sarstedt, Nümbrecht, Germany) blood sample was drawn. The EDTA S-Monovettes were centrifuged for 10 minutes at $350 x g$ and a temperature of $20^{\circ} \mathrm{C}$ (Centrifuge 5810 R, Eppendorf, Hamburg, Germany). After the centrifugation, $750 \mu$ plasma were transferred to Eppendorf Safe-Lock Tubes $(1.5 \mathrm{ml})$ and stored at $-80^{\circ} \mathrm{C}$ until further processing and testing.

\section{Clinical parameters / scores}

For each multiple trauma patient, the injury severity score (ISS) was calculated based on clinical findings, radiological examinations and intraoperative findings [29, 30]. In addition, the SIRS score was calculated on a daily basis. The SIRS score can range between 0 and 4 and is by definition positive for a SIRS for values $\geq 2$ [31]. Furthermore, the Denver multiple organ failure score, which detects a dysfunction of the lung, kidneys, liver and heart, was determined daily. The Denver MOF score can range between 0 and 12 . A value $>3$, which is calculated $48 \mathrm{~h}$ after the initial trauma, is defined as multiple organ failure [32-35].

\section{Enzyme-Linked Immunosorbent Assay (ELISA)}

The quantitative measurement of the adipokine plasma concentrations was performed with the following commercially available Enzyme-Linked Immunosorbent Assays (PeproTech, Hamburg, Germany): Human Leptin ELISA Development Kit (Catalog number: 900-K90 / Lot number: 0710090); Human Resistin Mini ELISA Development Kit (Catalog number: 900-M235 / Lot number: 0412235); Human IL-17A Mini ELISA Development Kit (Catalog number: 900-M84 / Lot number: 1212084-M); Human IL-33 Mini ELISA Development Kit (Catalog number: 900-M398 / Lot number: 1112398-M). All ELISAs were performed according to the manufacturer's manuals. The photometric measurement was performed with the ELISA Plate Reader FLUOstar Omega and the Reader Control Software (BMG Labtech, Ortenberg, Germany).

\section{Statistics}

The calculation of the adipokine concentrations from the OD values of the ELISAs, the statistical analysis of the data and the creation of the diagrams was performed with GraphPad PRISM Version 8.0.1 (GraphPad Software Inc., La Jolla, USA). The calculation of the individual adipokine concentrations from the OD values was carried out by an interpolation of the concentrations from the respectively generated standard curves. A normal distribution or logarithmic normal distribution of the data was tested with the D'Agostino-Pearson test (Omnibus K2). Comparisons between non-normally distributed values were 
analyzed with the non-parametric Mann-Whitney $U$ test. The analysis of the correlations between the nonnormally distributed values were performed with the non-parametric Spearman correlation. The applicability of adipokines as diagnostic markers for the discrimination between multiple trauma patients with and without MOF was analyzed with receiver operating characteristic curves (ROC curves). A significance level a of 0.05 was specified and the results were considered statistically significant at a pvalue of $<0.05$. The statistical analysis was checked for correctness by the Department of Statistics, Technical University of Munich.

\section{Results}

\section{Clinical parameters}

The values of the ISS of the individual multiple trauma patients ranged from 17-50, the median was 41. Of all included patients, $85.71 \%$ suffered a head injury (AIS $\geq 3$ ), $71.42 \%$ a chest trauma (AIS $\geq 3$ ) and $28.57 \%$ an abdominal trauma (AIS $\geq 3$ ). All 14 multiple trauma patients developed SIRS within $72 \mathrm{~h}$ after trauma, with an interindividual distinct degree of severity. On the first day, $100 \%$ of all multiple trauma patients fulfilled the criteria for SIRS, on the second day $46.15 \%$ and on the third and fourth day $50 \%$. In total, four patients suffered MOF according to the Denver MOF score with a value of $>3$ within three days after trauma. Three of those four patients had a maximum MOF score of 4 and one patient a maximum score of 5 . Nine patients, representing a total of $64.28 \%$ of all enrolled patients, died from their trauma sequelae within 18 days.

\section{Leptin}

The leptin plasma concentrations of the multiple trauma patients were statistically significantly higher at time point $0 \mathrm{~h}(\mathrm{p}$-value $=0.0148), 6 \mathrm{~h}(\mathrm{p}$-value $=0.0141), 24 \mathrm{~h}(\mathrm{p}$-value $=0.0023), 48 \mathrm{~h}(\mathrm{p}$-value $=0.0077)$ and $72 \mathrm{~h}$ ( $\mathrm{p}$-value $=0.0077$ ), as compared to the control group (Fig. 1A). The multiple trauma patients with MOF showed higher leptin concentrations at all five time points and at $72 \mathrm{~h}$, a statistically significantly ( $p$-value $=0.0283$ ) higher concentration compared to the multiple trauma patients without MOF (Fig. 1B).

The leptin concentration at the time point $72 \mathrm{~h}$ showed an area under the curve (AUC) of 0.9063 and a statistical significance with a p-value of 0.0272 .

\section{Resistin}

The resistin plasma concentrations of the multiple trauma patients were statistically significantly higher at time point $0 \mathrm{~h}(\mathrm{p}$-value $=0.0004), 6 \mathrm{~h}(\mathrm{p}$-value $=\leq 0.0001), 24 \mathrm{~h}(\mathrm{p}$-value $=\leq 0.0001), 48 \mathrm{~h}$ ( $\mathrm{p}$-value $\leq$ $0.0001)$ and $72 \mathrm{~h}$ ( $\mathrm{p}$-value $\leq 0.0001)$, as compared to the control group (Fig. 2).

There was a statistically significant positive correlation of the resistin concentrations of the multiple trauma patients at time point $24 \mathrm{~h}$ and the corresponding SIRS score on day 2 with an $r=0.5969$ (p-value $=0.0342$ ). In addition, there was a statistically significant positive correlation of resistin concentrations of 
the multiple trauma patients at $48 \mathrm{~h}$ and the corresponding SIRS score on day 3 with an $\mathrm{r}=0.5935(\mathrm{p}$ value $=0.0455$ ). Furthermore, there was a statistically significant positive correlation of the resistin concentrations of the multiple trauma patients at time point $0 \mathrm{~h}$ and the corresponding MOF score on day 2 with an $r=0.7283$ ( $p$-value $=0.0152)$.

\section{Interleukin-17A}

The interleukin-17A plasma concentrations of the multiple trauma patients were statistically significantly higher at time point $0 \mathrm{~h}(\mathrm{p}$-value $=0.0103), 6 \mathrm{~h}(\mathrm{p}$-value $=0.034), 24 \mathrm{~h}(\mathrm{p}$-value $=0.0016), 48 \mathrm{~h}(\mathrm{p}$-value $\leq$ $0.0001)$ and $72 \mathrm{~h}$ ( $\mathrm{p}$-value $\leq 0.0001)$, as compared to the control group (Fig. $3 \mathrm{~A})$. The multiple trauma patients with MOF showed statistically significantly higher interleukin-17A concentrations at time point $0 \mathrm{~h}$ ( $\mathrm{p}$-value $=0.0182), 48 \mathrm{~h}(\mathrm{p}$-value $=0.0485)$ and time point $72 \mathrm{~h}(\mathrm{p}$-value $=0.0238)$, as compared to the multiple trauma patients without MOF (Fig. 3B).

There was a statistically significant positive correlation of the interleukin-17A concentrations of the multiple trauma patients at time point $24 \mathrm{~h}$ and the corresponding SIRS scores on day 2 with an $\mathrm{r}=0.577$ ( $p$-value $=0.0418$ ) and on day 3 with an $r=0.5935$ ( $p$-value $=0.0455)$. In addition, there was a statistically significant positive correlation of the interleukin-17A concentrations of the multiple trauma patients at time point $6 \mathrm{~h}$ with an $r=0.7341(\mathrm{p}$-value $=0.010), 24 \mathrm{~h}$ with an $r=0.6478(\mathrm{p}$-value $=0.0292)$ and $48 \mathrm{~h}$ with an $r=0.6262(p$-value $=0.0363)$ and the corresponding SIRS score on day 4. Furthermore, there was a statistically significant positive correlation of the interleukin-17A concentrations of the multiple trauma patients at time point $48 \mathrm{~h}$ and the corresponding MOF score on day 2 with an $r=0.6199$ (p-value = 0.0356). The interleukin-17A concentration showed at time point $0 \mathrm{~h}$ an AUC of 0.963 and a statistical significance with a p-value of 0.0208 , at $48 \mathrm{~h}$ an AUC of 0.875 and a statistical significance with a p-value of 0.0415 and at time point $72 \mathrm{~h}$ an AUC of 0.9063 and a statistical significance with a p-value of 0.0272 .

\section{Interleukin-33}

The interleukin-33 plasma concentrations of the multiple trauma patients were statistically significantly higher at time point $6 \mathrm{~h}(\mathrm{p}$-value $=0.0319), 24 \mathrm{~h}(\mathrm{p}$-value $=0.0125), 48 \mathrm{~h}(\mathrm{p}$-value $=0.0007)$ and $72 \mathrm{~h}(\mathrm{p}$ value $=0.0003$ ) compared to the control group (Fig. 4).

There was a statistically significant positive correlation of the ISS and the corresponding interleukin-33 concentrations of the multiple trauma patients at time point $0 \mathrm{~h}$ with an $r=0.7425$ ( $p$-value $=0.0074$ ). Furthermore, there was a statistically significant positive correlation of the interleukin-33 concentrations of the multiple trauma patients at time point $0 \mathrm{~h}$ with an $r=0.6691$ ( $p$-value $=0.0390), 24 \mathrm{~h}$ with an $r=$ $0.6188(p$-value $=0.0355)$ and $48 \mathrm{~h}$ with an $r=0.6622(p$-value $=0.0223)$ and the corresponding SIRS score on day 3 . In addition, there was a statistically significant positive correlation of the interleukin-33 concentrations of the multiple trauma patients at time point $48 \mathrm{~h}$ with an $r=0.6478(p$-value $=0.0292)$ and $72 \mathrm{~h}$ with an $r=0.6262$ ( $p$-value $=0.0363$ ) and the corresponding SIRS score on day 4. Finally, there was a statistically significant positive correlation of the interleukin-33 concentrations of the multiple trauma patients at time point $0 \mathrm{~h}$ and the corresponding MOF score on day 3 with an $r=0.7967$ ( $p$-value $=$ 0.0089). 


\section{Discussion}

\section{Patients \& Methods}

The multiple trauma patient population of this study consisted of $57.1 \%$ men and $42.9 \%$ women, who had an average age of 49.7 years, while the patient population of the German TraumaRegister in the years 2012 to 2014 consisted of $68 \%$ men and $32 \%$ women, who had an average age of 57.0 years [36]. It was shown in both groups that the male sex prevails in the multiple trauma patient population, while our multiple trauma patients were younger on average. The multiple trauma patients of this study had a higher ISS with a median of 41, as compared to the TraumaRegister patients with an ISS of 29.1, who were thus more seriously injured [36]. In our study, $28.6 \%$ of the patients developed a MOF, which was comparable to the $32.7 \%$ in the TraumaRegister population between the years 2002 to 2011 [37]. In this work, the Denver MOF score was used since Grotz and coworkers showed in their work that the Denver score has a higher specificity ( $88 \%$ ) for the diagnosis of MOF than the Marshall score $(75 \%)$ and the Goris score (78\%) [34]. In addition, Hutchings and coworkers also recommend using the Denver MOF score as the gold standard for the diagnosis of multiple organ failure after trauma [38].

\section{Leptin}

In this study, the multiple trauma patients showed statistically significant higher leptin plasma concentrations at all five time points, as compared to the control group. In the study of Chachkhiani and coworkers, the patients who experienced injury in the form of visceral surgery, also had statistically significantly increased plasma concentrations of leptin at time point $24 \mathrm{~h}$, as compared to the preoperative values and to the healthy control group [39]. In this study, the multiple trauma patients with MOF showed higher leptin concentrations at all time points, as compared to the multiple trauma patients who did not develop MOF. In their study, Kimura and coworkers could show that patients who developed organ failure after a liver resection had significantly higher leptin concentrations [40]. The leptin concentration at time point $72 \mathrm{~h}$ was - according to the ROC curve analysis - a very good diagnostic marker for the discrimination between multiple trauma patients who developed multiple organ failure and the patients who did not. In the work of Yousef et al., leptin turned out to be a highly sensitive and specific marker for the discrimination between patients with SIRS or sepsis and patients without [41]. However, it should not be disregarded that other factors besides trauma have an influence on the leptin concentration. Thus, there is a significant correlation between the basal leptin concentration before surgery, which in turn correlates with BMI and peak leptin levels after surgery [42].

\section{Resistin}

The multiple trauma patients in this study showed statistically significantly higher resistin plasma concentrations at all five time points, as compared to the control group. Dong et al. revealed that patients with an isolated traumatic brain injury show significantly increased resistin plasma concentrations within the first 7 days after trauma [43]. In our study, there were statistically significant positive correlations between the resistin concentrations of the multiple trauma patients and the corresponding SIRS and MOF 
scores. Wade et al. found a significant positive correlation between the resistin plasma concentrations and the multiple organ dysfunction score in patients with burn trauma [44]. In addition, Sundén-Cullberg et al. demonstrated a statistically significant correlation between the resistin concentrations and the SOFA score in sepsis patients [45].

\section{Interleukin-17A}

The multiple trauma patients showed statistically significantly higher interleukin-17A plasma concentrations throughout the observation period, as compared to the control group. This is in line with the work of Abboud et al., who highlighted the importance of IL-17A in the post-traumatic immune response in patients with a severe blunt trauma [46]. Furthermore, Hefele et al. discovered an increased IL17A expression on $\mathrm{TH}-17$ cells and CD 4 + Tregs following trauma [47]. In this study, the multiple trauma patients with MOF showed statistically significantly higher IL-17A concentrations at the time points $0 \mathrm{~h}$, $48 \mathrm{~h}$ and $72 \mathrm{~h}$, as compared to the trauma patients without MOF. With the concordant findings of Abboud et al., IL-17A may play a role in the development of MOF [46]. In our study, there were statistically significant positive correlations between the IL-17A concentrations of the multiple trauma patients and the corresponding SIRS and MOF scores. In another study, however, no association was found between IL-17A levels and the multiple organ dysfunction score in multiple trauma patients [48]. This may possibly be explained by the fact that in that study only IL-17A concentrations at the day of admission to the intensive care unit were examined. In our study, the correlation of IL-17A and the MOF score was observed at $48 \mathrm{~h}$. According to the ROC curve analysis, the IL-17A concentrations at time points $0 \mathrm{~h}, 48 \mathrm{~h}$ and $72 \mathrm{~h}$ were very good diagnostic markers for the discrimination between multiple trauma patients who developed multiple organ failure and patients who did not. In the above discussed study from Abdelkader et al. it could be shown that multiple trauma patients with sepsis had elevated IL-17A concentrations and that IL-17A is a possible predictor of sepsis with an AUC of 0.687 [48].

\section{Interleukin-33}

The multiple trauma patients showed higher interleukin-33 concentrations at all five time points and at $6 \mathrm{~h}, 24 \mathrm{~h}, 48 \mathrm{~h}$ and $72 \mathrm{~h}$ statistically significantly higher concentrations, as compared to the control group. Furthermore, there was a statistically significant positive correlation of the ISS and the corresponding IL33 concentrations of the multiple trauma patients at time point $0 \mathrm{~h}$. These results are consistent with the function of IL-33 as an alarmin, which is released after cell damage in the context of multiple trauma [49]. Another study could demonstrate that trauma patients had elevated IL-33 concentrations in the first 7 days after severe blunt trauma [50]. However, there was no correlation between the IL-33 concentrations and the ISS [50]. This could be caused by the fact that these trauma patients were less seriously injured with a mean ISS of only 20.2 and the IL-33 concentration possibly shows a stronger correlation with higher ISS values. In our study, there were statistically significant positive correlations between the IL-33 concentrations of the multiple trauma patients and the corresponding SIRS and MOF scores. Xu et al. could demonstrate that trauma patients with an organ failure show elevated IL-33 levels [50].

\section{Conclusion}


The four adipokines investigated may play a role in the early posttraumatic immune response and the adipokines leptin, IL-17A and IL-33 in the development of SIRS and MODS. In addition, leptin and IL-17A may be useful as diagnostic biomarkers to identify multiple trauma patients at risk of developing multiple organ failure in an intensive care setting.

The insights gained in this study can help to identify patients with a developing multiple organ failure early within the framework of systemic inflammatory response syndrome after severe trauma, potentially leading to better treatment outcomes. Furthermore -as part of the Damage Control Surgery concept- the information of the adipokine concentrations could be included in the treatment planning. In this way, the decision on the invasiveness of the primary trauma care (damage control orthopedics vs. early total care) in risk patients can be made more profoundly in order to avoid a possible second hit. In addition, the adipokine levels could be used, not only for diagnosis and therapy planning, but for also for monitoring the success of surgical and intensive care. Lastly, the insights found in this study now need to be confirmed and validated in a multicenter study with a significantly larger number of multiple trauma patients.

\section{List Of Abbreviations}

AIS Abbreviated injury scale

AUC Area under the curve

BMI Body mass index

C Celsius

ELISA Enzyme-linked immunosorbent assay

EDTA Ethylenediaminetetraacetic acid

g Gravity

ISS Injury severity score

h Hour

IL Interleukin

$\mu$ Microliter

ml Milliliter

MODS Multiple organ dysfunction syndrome

MOF Multiple organ failure 
MT Multiple trauma

NF Nuclear factor

OD Optical density

ROC Receiver operating characteristic

SD Standard deviation

SIRS Systemic inflammatory response syndrome

TGF Transforming growth factor

TH T helper

TNF Tumor necrosis factor

\section{Declarations}

Ethics approval and consent to participate: Ethical committee approval was obtained from the local institutional review board of the University Munich (reference number: 012/00).

Signed informed consent was obtained from the participants, patients or legal guardians.

Consent for publication: Signed informed consent was obtained from the participants, patients or legal guardians.

Availability of data and materials: The datasets used and/or analysed during the current study are available from the corresponding author on reasonable request.

Competing interests: The authors declare that they have no competing interests.

Funding: Klinikum rechts der Isar, Technical University of Munich.

Authors' contributions: M.v.G., A.T.H. and J.H. designed the study; J.H., A.T.H., N.K. and M.U. performed the research; J.H. and A.T.H. performed the statistical analysis; J.H., A.T.H., M.v.G., V.B.-F., P.B. and M.H. analyzed and interpreted data; P.B. and M.H. provided critical clinical resources; J.H. and A.T.H. wrote the manuscript; all authors reviewed the draft manuscript and approved the final version for submission.

Acknowledgements: The authors thank Fritz Seidl, M.A. translating and interpreting, for editing and language revision of this manuscript.

\section{References}


1. Wick M, Ekkernkamp A, Muhr G. The epidemiology of multiple trauma. Der Chirurg; Zeitschrift fur alle Gebiete der operativen Medizen. 1997;68(11):1053-8.

2. Lefering R. Das schwere Trauma. Notfall+ Rettungsmedizin. 2008;11(6):373-6.

3. Ornato JP, Craren EJ, Nelson NM, Kimball KF. Impact of improved emergency medical services and emergency trauma care on the reduction in mortality from trauma. The Journal of trauma. 1985;25(7):575-9.

4. Baker CC. Epidemiology of trauma: the civilian perspective. Annals of emergency medicine. 1986;15(12):1389-91.

5. Regel G, Lobenhoffer P, Grotz M, Pape H, Lehmann U, Tscherne H. Treatment results of patients with multiple trauma: an analysis of 3406 cases treated between 1972 and 1991 at a German Level I Trauma Center. Journal of Trauma and Acute Care Surgery. 1995;38(1):70-8.

6. Keel M, Trentz O. Pathophysiology of polytrauma. Injury. 2005;36(6):691-709.

7. van Griensven M, Krettek C, Pape H-C. Immune Reactions afterTrauma. European Journal of Trauma. 2003;29(4):181-92.

8. Ouchi N, Parker JL, Lugus JJ, Walsh K. Adipokines in inflammation and metabolic disease. Nature reviews Immunology. 2011;11(2):85-97.

9. Berg AH, Scherer PE. Adipose Tissue, Inflammation, and Cardiovascular Disease. Circulation Research. 2005;96(9):939-49.

10. Javier C, Morena S, Rodolfo G, Verónica L, Jesús G-RJ, Francisca L, et al. Adipokines: Biofactors from white adipose tissue. A complex hub among inflammation, metabolism, and immunity. BioFactors. 2011;37(6):413-20.

11. Fantuzzi G, Faggioni R. Leptin in the regulation of immunity, inflammation, and hematopoiesis. Journal of leukocyte biology. 2000;68(4):437-46.

12. La Cava A, Matarese G. The weight of leptin in immunity. Nature reviews Immunology. 2004;4(5):3719.

13. Finck BN, Johnson RW. Tumor necrosis factor (TNF)-a induces leptin production through the p55 TNF receptor. American Journal of Physiology-Regulatory, Integrative and Comparative Physiology. 2000;278(2):R537-R43.

14. Santos-Alvarez J, Goberna R, Sánchez-Margalet V. Human leptin stimulates proliferation and activation of human circulating monocytes. Cellular immunology. 1999;194(1):6-11.

15. Negrin LL, Jahn A, van Griensven M. Leptin protects against mortality and organ dysfunction in a two-hit trauma/sepsis model and is IL-6-dependent. Shock. 2017;48(1):130-7.

16. Steppan CM, Brown EJ, Wright CM, Bhat S, Banerjee RR, Dai CY, et al. A family of tissue-specific resistin-like molecules. Proceedings of the National Academy of Sciences of the United States of America. 2001;98(2):502-6.

17. Patel L, Buckels AC, Kinghorn IJ, Murdock PR, Holbrook JD, Plumpton C, et al. Resistin is expressed in human macrophages and directly regulated by PPAR gamma activators. Biochemical and 
biophysical research communications. 2003;300(2):472-6.

18. Kaser S, Kaser A, Sandhofer A, Ebenbichler CF, Tilg H, Patsch JR. Resistin messenger-RNA expression is increased by proinflammatory cytokines in vitro. Biochemical and biophysical research communications. 2003;309(2):286-90.

19. Bokarewa M, Nagaev I, Dahlberg L, Smith U, Tarkowski A. Resistin, an adipokine with potent proinflammatory properties. Journal of immunology. 2005;174(9):5789-95.

20. Iwakura $Y$, Ishigame $H$, Saijo S, Nakae S. Functional specialization of interleukin-17 family members. Immunity. 2011;34(2):149-62.

21. Gaffen SL. An overview of IL-17 function and signaling. Cytokine. 2008;43(3):402-7.

22. Sabat R, Witte E, Witte K, Wolk K. IL-22 and IL-17: An Overview. In: Quesniaux V, Ryffel B, Padova F, editors. IL-17, IL-22 and Their Producing Cells: Role in Inflammation and Autoimmunity. Basel: Springer Basel; 2013. p. 11-35.

23. Mitsdoerffer M, Lee Y, Jäger A, Kim H-J, Korn T, Kolls JK, et al. Proinflammatory T helper type 17 cells are effective B-cell helpers. Proceedings of the National Academy of Sciences. 2010;107(32):142927.

24. Schmitz J, Owyang A, Oldham E, Song Y, Murphy E, McClanahan TK, et al. IL-33, an interleukin-1-like cytokine that signals via the IL-1 receptor-related protein ST2 and induces T helper type 2-associated cytokines. Immunity. 2005;23(5):479-90.

25. Wood IS, Wang B, Trayhurn P. IL-33, a recently identified interleukin-1 gene family member, is expressed in human adipocytes. Biochemical and biophysical research communications. 2009;384(1):105-9.

26. Lamkanfi M, Dixit VM. IL-33 raises alarm. Immunity. 2009;31(1):5-7.

27. Komai-Koma M, Xu D, Li Y, McKenzie AN, McInnes IB, Liew FY. IL-33 is a chemoattractant for human Th2 cells. European journal of immunology. 2007;37(10):2779-86.

28. Cherry WB, Yoon J, Bartemes KR, lijima K, Kita H. A novel IL-1 family cytokine, IL-33, potently activates human eosinophils. Journal of allergy and clinical immunology. 2008;121(6):1484-90.

29. Baker S, O'Neill B, Haddon Jr W, Long WB. The injury severity score: a method for describing patients with multiple injuries and evaluating emergency care. Journal of Trauma and Acute Care Surgery. 1974;14(3):187-96.

30. Baker S, O'Neill B. The injury severity score: an update. Journal of Trauma and Acute Care Surgery. 1976;16(11):882-5.

31. Bone RC, Balk RA, Cerra FB, Dellinger RP, Fein AM, Knaus WA, et al. Definitions for sepsis and organ failure and guidelines for the use of innovative therapies in sepsis. Chest. 1992;101(6):1644-55.

32. Sauaia A, Moore EE, Johnson JL, Ciesla DJ, Biffl WL, Banerjee A. Validation of postinjury multiple organ failure scores. Shock (Augusta, Ga). 2009;31(5):438.

33. Sauaia A, Moore FA, Moore EE, Lezotte DC. Early risk factors for postinjury multiple organ failure. World journal of surgery. 1996;20(4):392-400. 
34. Grotz M, van Griensven M, Stalp M, Kaufmann U, Hildebrand F, Pape H. Scoring des Multiorganversagens nach schwerem Trauma Vergleich des Goris-, Marshall-und Moore-Scores. Der Chirurg. 2001;72(6):723-30.

35. Dewar D, Moore FA, Moore EE, Balogh Z. Postinjury multiple organ failure. Injury. 2009;40(9):912-8.

36. Sektion Notfall- \& Intensivmedizin, Schwerverletztenversorgung (NIS) der Deutschen Gesellschaft für Unfallchirurgie (DGU). TraumaRegister Jahresbericht 20152015.

37. Fröhlich $M$, Lefering R, Probst $C$, Paffrath $T$, Schneider MM, Maegele $M$, et al. Epidemiology and risk factors of multiple-organ failure after multiple trauma: an analysis of 31,154 patients from the TraumaRegister DGU. Journal of Trauma and Acute Care Surgery. 2014;76(4):921-8.

38. Hutchings L, Watkinson P, Young JD, Willett K. Defining multiple organ failure after major trauma: a comparison of the Denver, Sequential Organ Failure Assessment and Marshall scoring systems. The journal of trauma and acute care surgery. 2017;82(3):534.

39. Chachkhiani I, Gurlich R, Maruna P, Frasko R, Lindner J. The postoperative stress response and its reflection in cytokine network and leptin plasma levels. Physiol Res. 2005;54(3):279-85.

40. Kimura F, Shimizu H, Yoshidome H, Ohtsuka M, Kato A, Yoshitomi H, et al. Circulating cytokines, chemokines, and stress hormones are increased in patients with organ dysfunction following liver resection. Journal of Surgical Research. 2006;133(2):102-12.

41. Yousef AAA-M, Amr YM, Suliman GA. The diagnostic value of serum leptin monitoring and its correlation with tumor necrosis factor-a in critically ill patients: a prospective observational study. Critical care. 2010;14(2):R33.

42. Wallace AM, Sattar N, Mcmillan DC. The co-ordinated cytokine/hormone response to acute injury incorporates leptin. Cytokine. 2000;12(7):1042-5.

43. Dong X-Q, Yang S-B, Zhu F-L, Lv Q-W, Zhang G-H, Huang H-B. Resistin is associated with mortality in patients with traumatic brain injury. Critical care. 2010;14(5):R190.

44. Wade CE, Mora AG, Shields BA, Pidcoke HF, Baer LA, Chung KK, et al. Signals from fat after injury: plasma adipokines and ghrelin concentrations in the severely burned. Cytokine. 2013;61(1):78-83.

45. Sundén-Cullberg J, Nyström T, Lee ML, Mullins GE, Tokics L, Andersson J, et al. Pronounced elevation of resistin correlates with severity of disease in severe sepsis and septic shock. Critical care medicine. 2007;35(6):1536-42.

46. Abboud A, Namas RA, Ramadan M, Mi Q, Almahmoud K, Abdul-Malak O, et al. Computational analysis supports an early, type 17 cell-associated divergence of blunt trauma survival and mortality. Critical care medicine. 2016;44(11):e1074.

47. Hefele F, Ditsch A, Krysiak N, Caldwell C, Biberthaler P, van Griensven M, et al. Trauma Induces Interleukin-17A Expression on Th17 Cells and CD4+ Regulatory T Cells as Well as Platelet Dysfunction. Frontiers in immunology. 2019;10:2389.

48. Abdelkader A, El-Sayed M, Eladawy A, Elsayed R, Mukhtar A, Hammimy W. Interleukin-17a as a predictor of occurrence of sepsis in polytrauma patients: a prospective observational study. Intensive care medicine experimental. 2015;3(1):A790. 
49. Hirsiger S, Simmen H-P, Werner CM, Wanner GA, Rittirsch D. Danger signals activating the immune response after trauma. Mediators of inflammation. 2012;2012.

50. Xu J, Guardado J, Hoffman R, Xu H, Namas R, Vodovotz Y, et al. IL33-mediated ILC2 activation and neutrophil IL 5 production in the lung response after severe trauma: A reverse translation study from a human cohort to a mouse trauma model. PLoS medicine. 2017;14(7):e1002365.

Figures 
A

\section{Leptin}

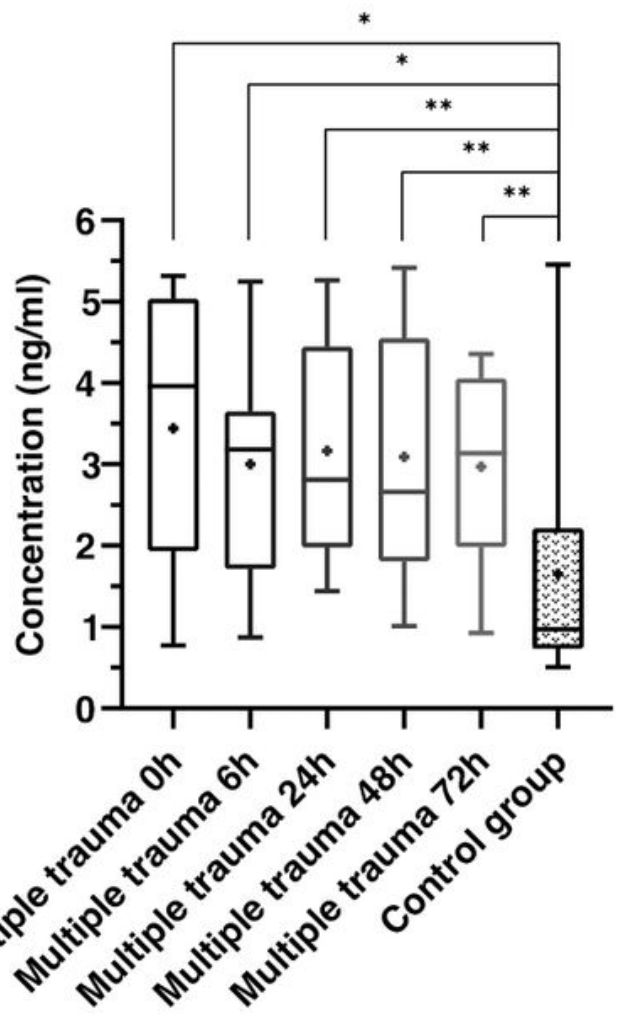

B

Leptin

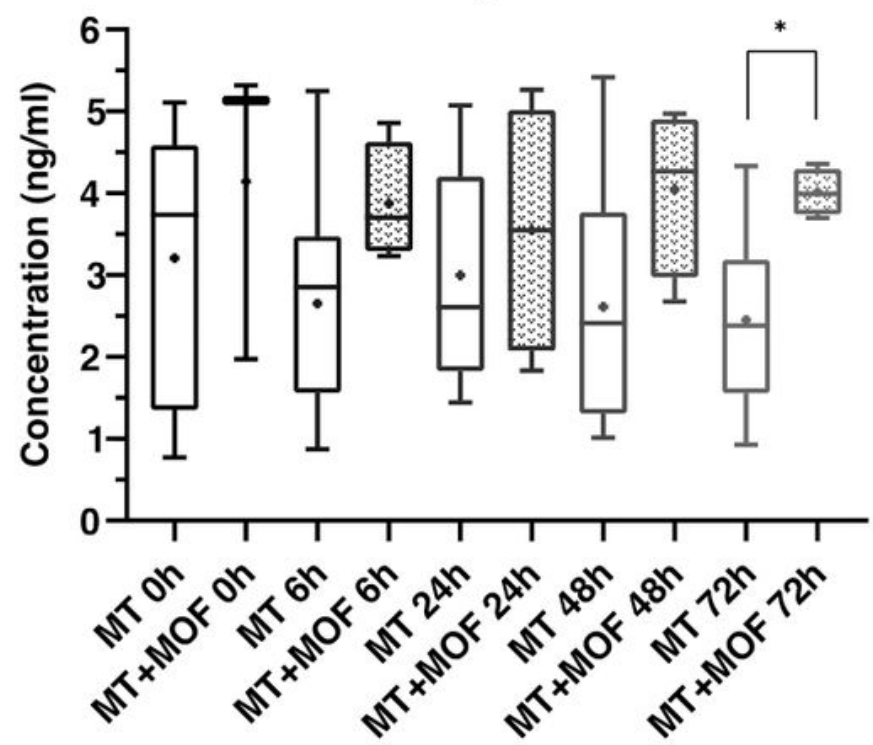

Figure 1

A Comparison of leptin plasma concentrations at the individual time points between multiple trauma patients and control group. Depiction of the concentrations as box-and-whisker plots with minimum / maximum value and + depicting the mean value. Statistical analysis of the data was performed by MannWhitney U test $(*=p<0.05 ; * \star=p<0.01)$. nmultiple trauma $0 h=12$, nmultiple trauma $6 h=14$, nmultiple trauma $24 \mathrm{~h}=13$, nmultiple trauma $48 \mathrm{~h}=12$, nmultiple trauma $72 \mathrm{~h}=12$, ncontrol group $=14 \mathrm{~B}$ Comparison of 
leptin plasma concentrations at the individual time points between multiple trauma patients without (MT) and multiple trauma patients with multiple organ failure (MT+MOF). Depiction of the concentrations as box-and-whisker plots with minimum / maximum value and + depicting the mean value. Statistical analysis of the data was performed by Mann-Whitney U test $\left({ }^{*}=p<0.05\right)$. nMT $0 h=9, n M T+M O F O h=3$, nMT 6h=10, nMT+MOF 6h=4, nMT 24h=9, nMT+MOF 24h=4, nMT 48h=8, nMT+MOF 48h=4, nMT 72h=8, $\mathrm{nMT}+\mathrm{MOF} 72 \mathrm{~h}=4$

Resistin

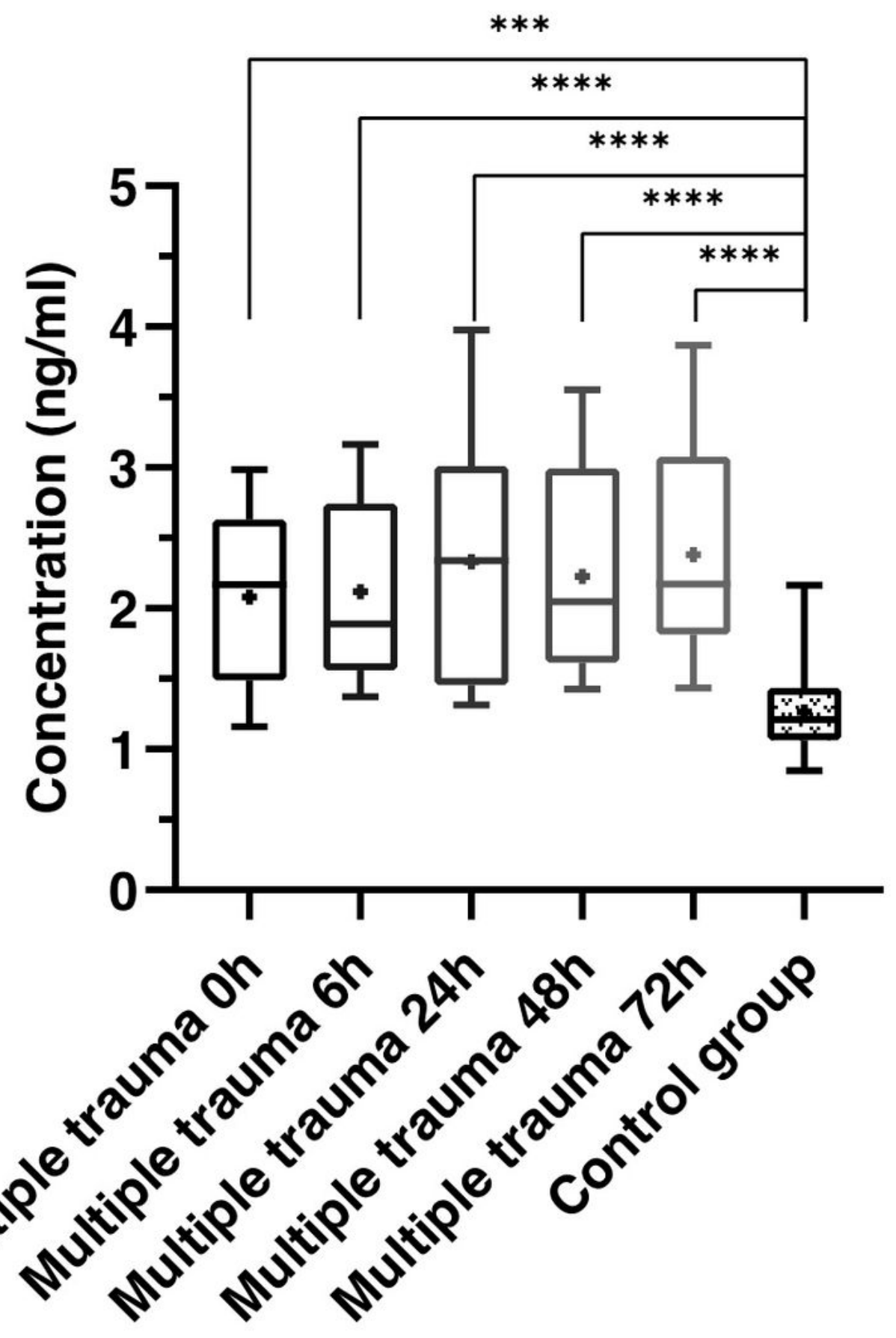

Figure 2 
Comparison of resistin plasma concentrations at the individual time points between multiple trauma patients and control group. Depiction of the concentrations as box-and-whisker plots with minimum / maximum value and + depicting the mean value. Statistical analysis of the data was performed by MannWhitney U test $(* \star \star=p<0.001 ; * \star \star *=p \leq 0.0001)$. nmultiple trauma $0 h=12$, nmultiple trauma $6 h=14$, nmultiple trauma $24 \mathrm{~h}=13$, nmultiple trauma $48 \mathrm{~h}=12$, nmultiple trauma $72 \mathrm{~h}=12$, ncontrol group=14

A

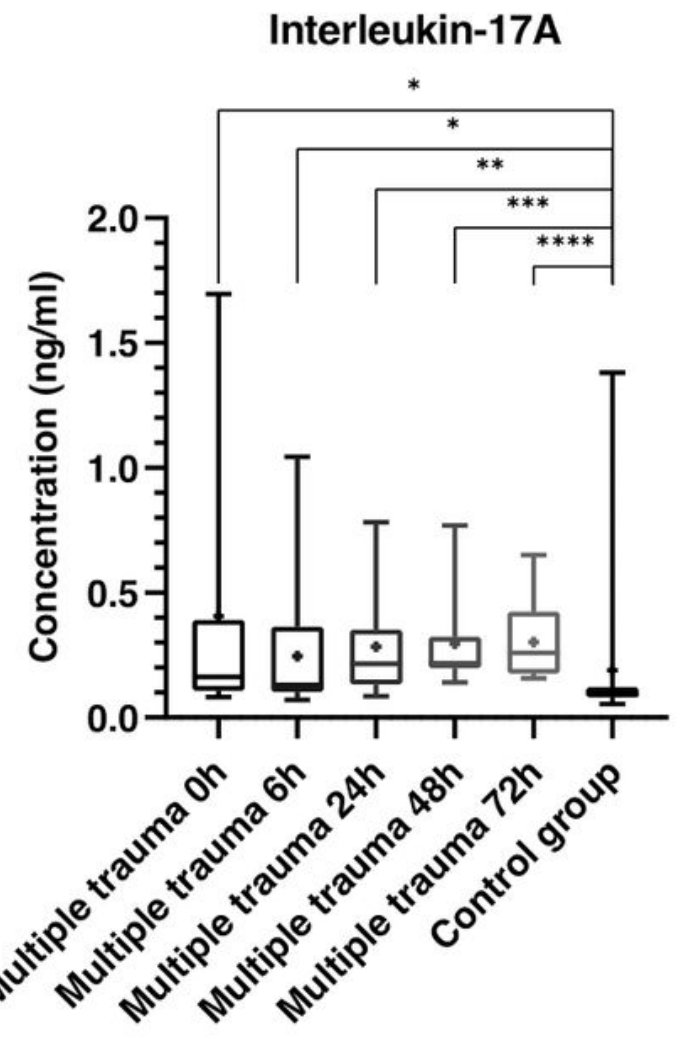

B

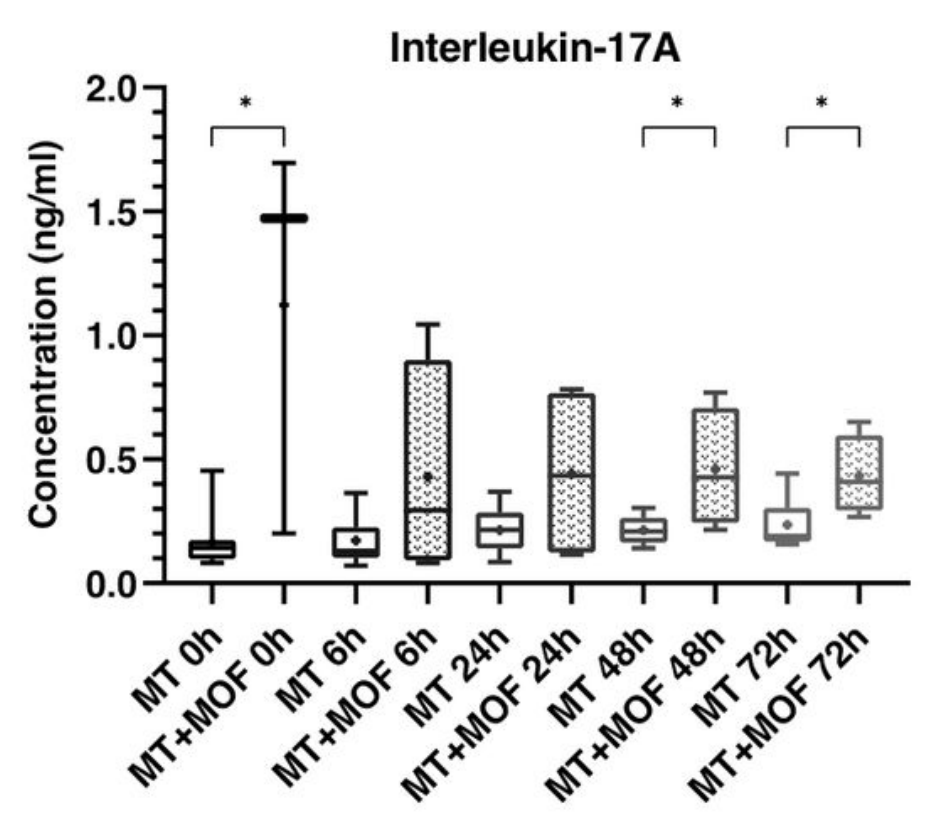

Figure 3 
A Comparison of interleukin-17A plasma concentrations at the individual time points between multiple trauma patients and control group. Depiction of the concentrations as box-and-whisker plots with minimum / maximum value and + depicting the mean value. Statistical analysis of the data was performed by Mann-Whitney U test $\left({ }^{*}=p<0.05 ;{ }^{* *}=p<0.01 ; * \star *=p<0.001 ; * \star \star *=p \leq 0.0001\right)$. nmultiple trauma $0 \mathrm{~h}=12$, nmultiple trauma $6 \mathrm{~h}=14$, nmultiple trauma $24 \mathrm{~h}=13$, nmultiple trauma $48 \mathrm{~h}=12$, nmultiple trauma $72 \mathrm{~h}=12$, ncontrol group=14 B Comparison of interleukin-17A plasma concentrations at the individual time points between multiple trauma patients without (MT) and multiple trauma patients with multiple organ failure (MT+MOF). Depiction of the concentrations as box-and-whisker plots with minimum / maximum value and + depicting the mean value. Statistical analysis of the data was performed by Mann-Whitney $U$ test $\left({ }^{*}=p<0.05\right) . n M T 0 h=9, n M T+M O F 0 h=3, n M T 6 h=10, n M T+M O F \quad 6 h=4, n M T 24 h=9, n M T+M O F$ $24 \mathrm{~h}=4, \mathrm{nMT} 48 \mathrm{~h}=8, \mathrm{nMT}+\mathrm{MOF} 48 \mathrm{~h}=4, \mathrm{nMT} 72 \mathrm{~h}=8, \mathrm{nMT}+\mathrm{MOF} 72 \mathrm{~h}=4$ 


\section{Interleukin-33}
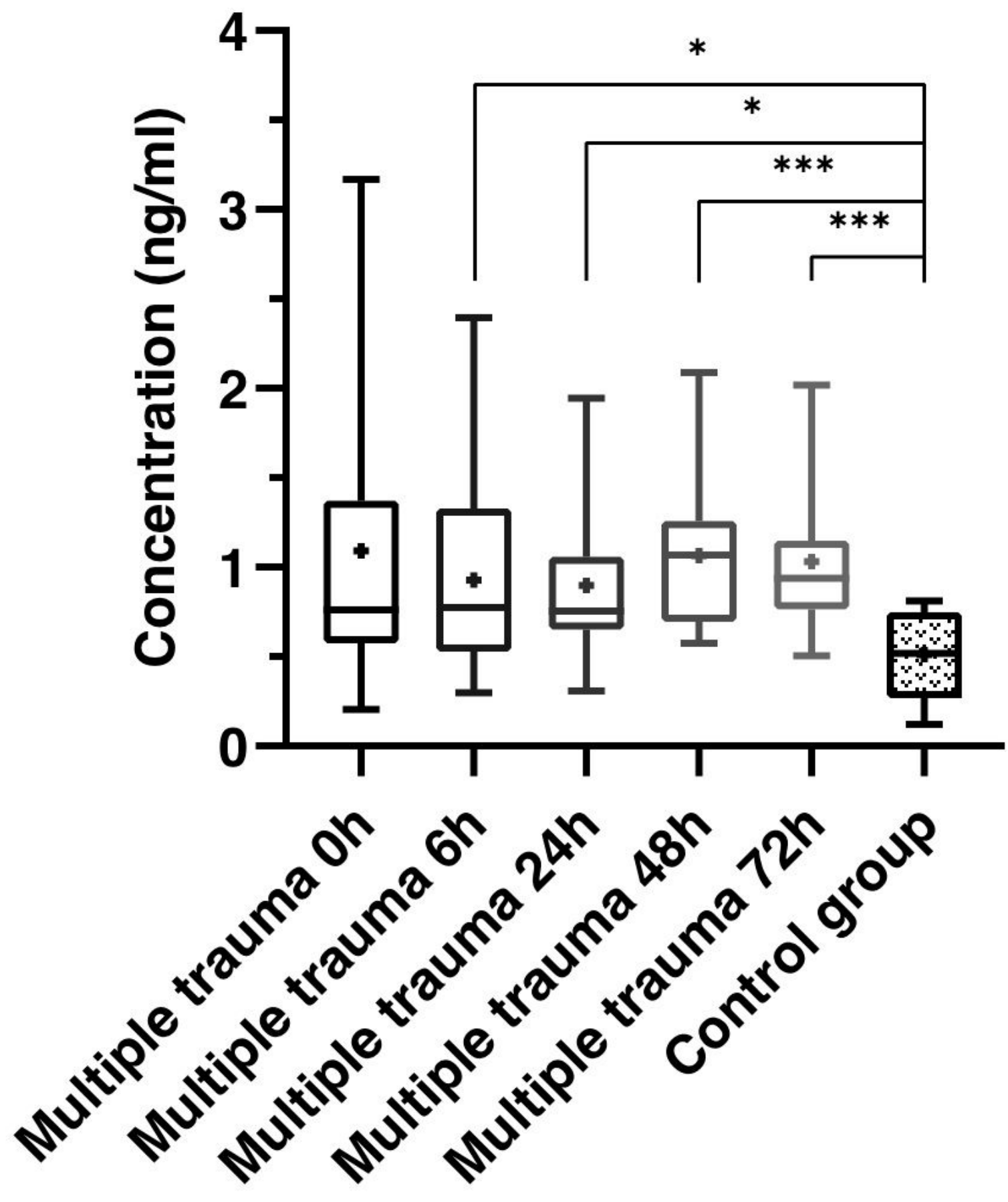

Figure 4

Comparison of interleukin-33 plasma concentrations at the individual time points between multiple trauma patients and control group. Depiction of the concentrations as box-and-whisker plots with minimum / maximum value and + depicting the mean value. Statistical analysis of the data was performed by Mann-Whitney U test $(*=p<0.05 ; * \star *=p<0.001)$. nmultiple trauma $0 \mathrm{~h}=12$, nmultiple trauma $6 \mathrm{~h}=14$, nmultiple trauma $24 \mathrm{~h}=13$, nmultiple trauma $48 \mathrm{~h}=12$, nmultiple trauma $72 \mathrm{~h}=12$, ncontrol group $=14$ 